\title{
DESCRIPTION AND ANALYSIS OF INTERVAL SYSTEMS
}

\author{
Radek Matušů, Roman Prokop and Jiří Korbel \\ Faculty of Applied Informatics \\ Tomas Bata University in Zlín \\ Nad Stráněmi 4511, 76005 Zlín, Czech Republic \\ E-mail: \{rmatusu; prokop; korbel\}@fai.utb.cz
}

\begin{abstract}
KEYWORDS
Interval Uncertainty, Robust Stability, Kharitonov Theorem, Value Set Concept, Zero Exclusion Condition.
\end{abstract}

\begin{abstract}
Control of real industrial processes is almost always burden with an uncertainty. The reasons of that unfavorable fact are mainly an imprecise modelling and measuring or an influence of some external conditions. A convenient tool for dealing with such class of issues represents the application of models with parametric uncertainty. The mathematical model is then supposed to contain parameters which are not precisely known, but the values thereof lie within given intervals. If individual uncertain coefficients (in polynomial, in transfer function etc.) are mutually independent, the uncertainty has a simple interval structure. This contribution offers several possibilities of utilization of interval uncertainty for systems description and presents the tools for robust stability analysis, emphasizing advantages and limitations connected with the use of this simple structure, even for more complicated problems.
\end{abstract}

\section{INTRODUCTION}

During the second half of the last century, the one of thorny problems emerged in theory of automatic control. New analytical methods of synthesis, which assume an exact mathematical model of the process to be controlled, paid insufficient attention to uncertainty caused by imperfections in modelling or changeable physical properties of the plant. On that account, uncertain systems have become very attractive from the point of view of scientific research and also applications during previous almost three decades. It is fully natural, that number of approaches that solve the related problems has been developed and improved during this long-term interest.

The classical and probably also the most required task is to ensure appropriate control of the system which is affected by some changes, variations, perturbations or disturbances. Generally, more basic principles how to solve these issues exist. A relatively clear and comprehensible idea is incorporated in robust control. Its aim is to design the one fixed controller which guarantees suitable behaviour not only for nominal system, but also for some neighbourhood. Robust controllers are preferred in contemporary practice, simple and easily utilizable. On the contrary, the abilities of these compensators are limited at great or fast changes in controlled system or operating conditions and, furthermore, robust control responses need not to "optimal" for all supposed states.

This contribution is focused mainly on problems of description and analysis of systems with interval uncertainty and their importance in theory and practice of process control. Interval uncertainty and related tools for robust stability testing represent essentials which many authors draw upon it during the research of analysis and synthesis methods for more complicated parametric uncertainties and moreover, the theory is also quite well applicable for practical robust control problems. First, the paper outlines the motivation for use of uncertain mathematical model and provides the categorization of basic robustness terms. Further, it is shown a possible notation of interval polynomials and several tools for analytical and graphical testing of robust stability (such as Kharitonov theorem, the value set concept and the zero exclusion condition) are presented. Finally, the possible utilization of the interval uncertainty theory for more complicated uncertainty structures is discussed.

\section{MATHEMATICAL MODEL WITH UNCERTAINTY}

Most of the technological processes can be modelled as linear time-invariant systems, in spite of the fact, that their real behaviour is often different and much more complex. The motivation is evident - owing to this, the transfer functions can be used for description of such systems and subsequently also the control theory of linear systems, which is very well-developed, can be applied. However, an effort to create the simple enough model almost always leads to the origin of uncertainty. Their emergence often consists in neglect of "less important properties", especially from the realms of fast dynamic effects, nonlinearities or time-variant behaviours of the plant.

Nevertheless, the presence of uncertainty can not be excluded even if the processes are in essence linear, because, strictly speaking, the physical parameters are never exactly known, possibly they can vary according to operating conditions. Ergo the principal problem is, if the controller designed for nominal system is able to keep some properties of the feedback control loop also for really controlled system, which falls into certain 
neighbourhood. In other words, if the controller can keep these properties not only for one nominal system, but for the whole family of systems (Kučera 2001).

The uncertainty in constructed mathematical model and thus the size of neigbourhood which should the controller cope with can be taken into consideration and described in the two main ways - as parametric or nonparametric uncertainty. The former, nonparametric description of uncertainty lies in restriction of area of possible appearance of frequency characteristic. It is associated with unmodelled dynamics, truncation of high frequency modes, nonlinearities, randomness in the systems, etc. The latter, parametric approach then represents known structure but uncertain knowledge of actual physical parameters of the controlled system. Their possible values are usually bounded by intervals. This work deals mainly with interval systems, that is one class of systems with parametric uncertainty.

The systems with parametric uncertainty are often described via a vector of real uncertain parameters $q$. In a simplified way, vector $q$ is called the uncertainty.

The very frequent subject of investigation is the uncertain characteristic polynomial of the closed-loop control system. Assume that this polynomial is described by:

$$
p(s, q)=\sum_{i=0}^{n} \rho_{i}(q) s^{i}
$$

where $\rho_{i}$ are coefficient functions.

In robustness problems, the vector of uncertain parameters $q$ is usually supposed to be confined by the uncertainty bounding set $Q$, which is given a priory (from identification, by user requirements, etc.). The set $Q$ is taken to be a ball in some appropriate norm. Combination of the polynomial (1) with its uncertainty bounding set $Q$ constitutes a family of polynomials.

The robustness issues consist in several tasks. The problem investigated in this contribution is robust stability analysis. Generally, suppose that every member of family $F$ (family of polynomials) has a property $S$ (stability) - the family $F$ is then designated as robust (robustly stable).

It is well-known that the continuous-time polynomial $p(s)$ is stable if and only if all its roots lie in the left half of the complex plane or, in other words, if and only if all its roots have negative real part. The general family of polynomials $P=\{p(\cdot, q): q \in Q\}$ is robustly stable if and only if $p(\cdot, q)$ is stable for all $q \in Q$, i.e. all roots of $p(s, q)$ must be located in the left complex half plane for all $q \in Q$.

The uncertainty enters into the polynomial (1) through the coefficient functions $\rho_{i}(q)$. And the way how the uncertain parameters enter into the coefficients of this polynomial is very significant. In accordance with this, several basic structures of uncertainty with increasing generality are distinguished:

- independent (interval) uncertainty structure
- affine linear uncertainty structure

- multilinear uncertainty structure

- nonlinear uncertainty structure (polynomial, general)

Moreover, the single parameter uncertainty is considered as a special case.

This paper deals deeply only with the case of $Q$ in the shape of box (which corresponds $L_{\infty}$ norm) and independent uncertainty structure.

\section{INTERVAL UNCERTAINTY}

The basic case of an uncertainty structure is the interval uncertainty. The essential condition is the independence of its structure. An uncertain polynomial (1) is said to have an independent uncertainty structure if each component $q_{i}$ of $q$ enters into only one coefficient. A family of polynomials:

$$
P=\{p(\cdot, q): q \in Q\}
$$

is called an interval polynomial family (or an interval polynomial for short) if it has an independent uncertainty structure, each coefficient depends continuously on $q$ and $Q$ is a box. The examples are:

$$
\begin{aligned}
& p_{1}(s, q)=\left(3+q_{1}\right) s^{2}+\left(5+q_{2}\right) s+\left(2+q_{3}\right) \\
& \quad q_{1} \in\langle 0.5 ; 2\rangle, q_{2} \in\langle-1 ; 3\rangle, q_{3} \in\langle 1 ; 2\rangle \\
& p_{2}(s, q)=s^{2}+\left(8+3 q_{1}+2 q_{2}\right) s+\left(5+2 q_{3}-q_{4}\right) \\
& \left|q_{i}\right| \leq 1
\end{aligned}
$$

Such a representation of the interval uncertainty relatively often involves a redundancy - see the second polynomial (4). Therefore, simplified notation in which each uncertain coefficient is expressed only as an interval is used instead. The new family is sometimes called a lumped version of the original one. For example, the uncertainty incorporated in (4) can be easily "lumped" by defining of new uncertain parameters:

$$
\begin{aligned}
& \tilde{q}_{1}=8+3 q_{1}+2 q_{2} \\
& \tilde{q}_{0}=5+2 q_{3}-q_{4}
\end{aligned}
$$

with respective new uncertainty bounding set:

$$
\tilde{q}_{1} \in\langle 3 ; 13\rangle ; \quad \tilde{q}_{0} \in\langle 2 ; 8\rangle
$$

and the new uncertain polynomial:

$$
\tilde{p}(s, \tilde{q})=s^{2}+\tilde{q}_{1} s+\tilde{q}_{0}
$$

Naturally, the resulting polynomial is shortly written as:

$$
\tilde{p}(s, \tilde{q})=s^{2}+[3 ; 13] s+[2 ; 8]
$$

Generally speaking, the ordinarily used shorthand notation of an interval polynomial looks like:

$$
p(s, q)=\sum_{i=0}^{n}\left[q_{i}^{-} ; q_{i}^{+}\right] s^{i}
$$




\section{THE KHARITONOV THEOREM}

The veritable milestone in robust stability analysis of systems under parametric uncertainty has become the Kharitonov theorem (Kharitonov 1979). Even though it was published in a Russian differential equations journal as early as in 1978, it has been "discovered" for the control community by Barmish (Barmish 1983; Barmish 1994) and also Bialas (Bialas 1983) entire four years later. This fundamental theorem says that an interval polynomial family with invariant degree is stable if and only if its four Kharitonov polynomials are stable. Thus, it is not necessary to check the stability of all possible extreme variations (which is $2^{l}$ if $q \in \mathbf{R}^{l}$ ), but always only of four polynomials without regard to the number of uncertain parameters. It is obvious that this reduction is of immense significance. The construction of Kharitonov polynomials is very simple and based on special fixed sequence of upper and lower bounds of coefficients in interval polynomial (9) according to the scheme:

$$
\begin{aligned}
& K_{1}(s)=q_{0}^{-}+q_{1}^{-} s+q_{2}^{+} s^{2}+q_{3}^{+} s^{3}+\cdots \\
& K_{2}(s)=q_{0}^{+}+q_{1}^{+} s+q_{2}^{-} s^{2}+q_{3}^{-} s^{3}+\cdots \\
& K_{3}(s)=q_{0}^{+}+q_{1}^{-} s+q_{2}^{-} s^{2}+q_{3}^{+} s^{3}+\cdots \\
& K_{4}(s)=q_{0}^{-}+q_{1}^{+} s+q_{2}^{+} s^{2}+q_{3}^{-} s^{3}+\cdots
\end{aligned}
$$

The application of Kharitonov theorem illustrates the next example. The task is to decide on the stability of the interval polynomial:

$$
p(s, q)=[0.5 ; 1.5] s^{3}+[3 ; 4] s^{2}+[1 ; 2] s+[0.5 ; 1]
$$

All four Kharitonov polynomials:

$$
\begin{aligned}
& K_{1}(s)=0.5+1 s+4 s^{2}+1.5 s^{3} \\
& K_{2}(s)=1+2 s+3 s^{2}+0.5 s^{3} \\
& K_{3}(s)=1+1 s+3 s^{2}+1.5 s^{3} \\
& K_{4}(s)=0.5+2 s+4 s^{2}+0.5 s^{3}
\end{aligned}
$$

are stable and hence the original interval polynomial (11) is also stable.

An advantageous software environment for robust stability analyis of systems with parametric uncertainty represents Polynomial Toolbox for Matlab (Polyx 2009; Sebek et al. 2000). The Kharitonov polynomials can be constructed in the Polynomial Toolbox via the command "kharit".

The original Kharitonov's proof of his theorem is complicated and relatively hard to understand. Substantially simpler geometric proofs can be found e.g. in (Anagnost et al. 1989; Dasgupta 1988; Minnichelli et al. 1989) or in (Matušů and Prokop 2008).

Besides, an array of improvements of the Kharitonov theorem can be found in literature. Among the most frequent ones the following are counted: the simplification for low-degree polynomials (e.g. for the polynomial degree $n=3$ and $q_{0}^{-}>0$ to check $K_{3}$ is enough) (Anderson et al. 1987); extensions with degree dropping (Barmish 1994); an alternative technique of robust stability investigation in a form of positivity condition of the frequency sweeping function (Barmish 1989); or Kharitonov's generalization of his theorem for an interval polynomials with complex coefficients (Barmish 1994; Kharitonov 1979).

\section{KHARITONOV RECTANGLES}

The Kharitonov rectangles are special kind of the value set, which will be generally defined later and which plays the essential role in solving robust stability problems. Suppose an interval polynomial (9) and substitute the complex variable $s$ for $j \omega$ with a fixed real frequency $\omega_{0}$. The value set of interval polynomial is then a two-dimensional set of all possible complex values which arise in complex plane letting $q$ range over the $Q$, i.e. letting all coefficients range over their intervals. Such a value set is always of a rectangular shape (rarely line segment as a special degraded case) and its sides are parallel to the axis. As mentioned above, this value set is called Kharitonov rectangle for the frequency $\omega_{0}$.

The fig. 1 shows the Kharitonov rectangle of interval polynomial (11) for frequency $\omega_{0}=0.2$ with highlighted vertexes.

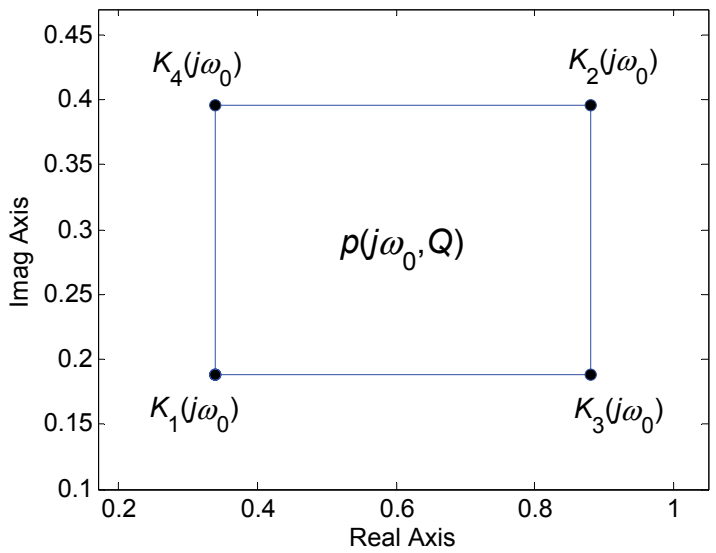

Figure 1: Kharitonov rectangle of interval polynomial (11) for $\omega_{0}=0.2$

Increasing of $\omega$ results in motion of the Kharitonov rectangle, i.e. a rectangle moves around the complex plane - see fig. 2, where Kharitonov rectangles for interval polynomial (11) are depicted (with the assistance of the Polynomial Toolbox command "khplot") for frequencies $\omega \in\langle 0 ; 1\rangle$ in the range of 50 samples. 


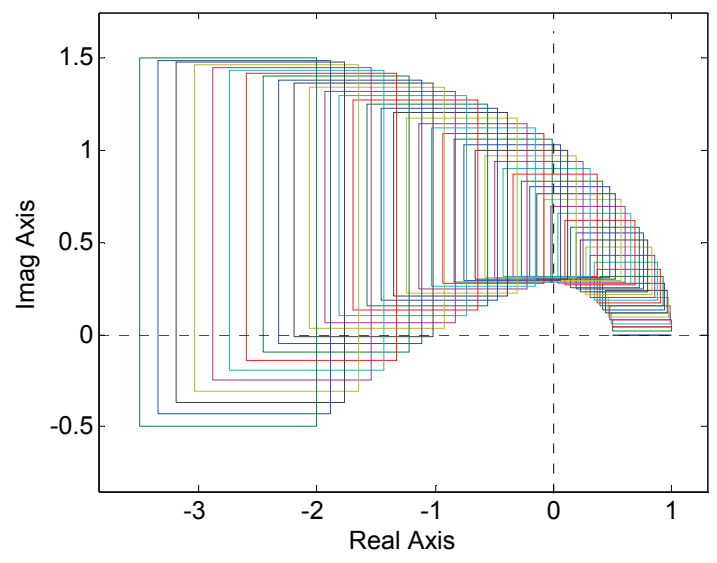

Figure 2: Kharitonov rectangles of interval polynomial (11) for $\omega \in\langle 0 ; 1\rangle$

\section{GRAPHICAL TEST OF ROBUST STABILITY}

An interval polynomial family $P=\{p(s, q): q \in Q\}$ with invariant degree and at least one stable member $p\left(s, q^{0}\right)$ is robustly stable if and only if the Kharitonov rectangles are excluded from the origin of the complex plane at all nonnegative frequencies, that is $0 \notin p(j \omega, Q) \quad \forall \omega \geq 0$. This rule, known as the zero exclusion condition, is very important, because in conjunction with the value set concept it represents powerful and sometimes practically the one and only conceivable method of robust stability analysis for much more complicated uncertainty structures (the coefficients must be continuous functions on given intervals). The usage of this test is essentially superfluous in the case of interval uncertainty, because the Kharitonov theorem is simpler, nevertheless the main ideas can be easily graphically verified and demonstrated - see e.g. fig. 2, where the interval polynomial family (11) contains a stable member and the zero point is excluded from the Kharitonov rectangles and hence the family is robustly stable. If the rectangles encompass the origin, it is apparent that the family will harbour the polynomial with root on imaginary axis (the stability border) - remember also the classical stability testing of polynomials via the Mikhailov(-Leonhard) criterion (Mikhailov 1938). The example of robustly unstable interval polynomial can be:

$$
\begin{aligned}
& p(s, q)=s^{5}+[3 ; 5] s^{4}+[5.5 ; 10] s^{3}+[6.5 ; 10] s^{2}+ \\
& +[3 ; 5] s+[0.5 ; 1]
\end{aligned}
$$

The Kharitonov rectangles, plotted again in the Polynomial Toolbox (for frequencies $\omega \in\langle 0 ; 3\rangle$ with the step 0.015), for this once cross the zero as shown in fig. 3 and fig. 4, while the second graph is zoomed to see better what is happening in the neighbourhood of the point $[0 ; 0 i]$. Therefore, the interval polynomial (13) is not robustly stable. In addition, it can be readily confirmed by the stability of only three of four Kharitonov polynomials - the unstable one is $K_{2}(s)$.

$$
\begin{aligned}
& K_{1}(s)=0.5+3 s+10 s^{2}+10 s^{3}+3 s^{4}+s^{5} \\
& K_{2}(s)=1+5 s+6.5 s^{2}+5.5 s^{3}+5 s^{4}+s^{5} \\
& K_{3}(s)=1+3 s+6.5 s^{2}+10 s^{3}+5 s^{4}+s^{5} \\
& K_{4}(s)=0.5+5 s+10 s^{2}+5.5 s^{3}+3 s^{4}+s^{5}
\end{aligned}
$$

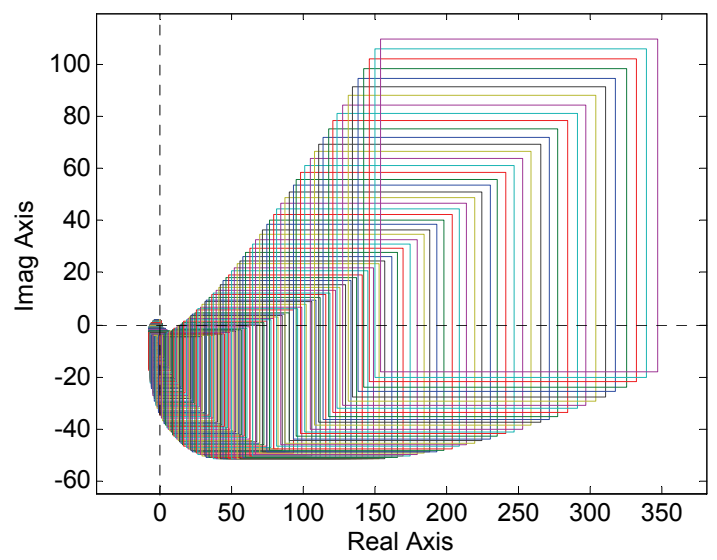

Figure 3: Kharitonov rectangles of interval polynomial (13) for $\omega \in\langle 0 ; 3\rangle$

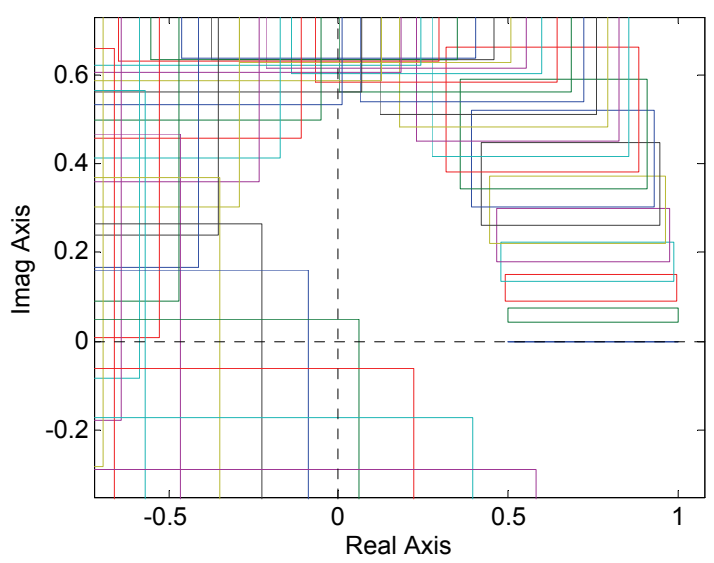

Figure 4: Kharitonov rectangles of interval polynomial (13) - detailed view

Furthermore, suggested simple graphical procedure for checking robust stability can be improved by finding of some finite precomputable frequency (so-called cutoff frequency) at which the frequency sweep can be terminated, i.e. the graphical test concluded (Barmish 1994).

\section{THE OVERBOUNDING METHOD}

However, it remains true that the interval uncertainty structure is considerably idealized and restrictive state because uncertain parameters typically enter into more than the only one coefficient (e.g., even an interval 
system in closed control loop leads, except for some specific cases, to the closed-loop characteristic polynomial with affine linear structure of uncertainty). In spite of that, the tools for robust stability analysis of interval systems can be utilized also for these more general events as an alternative to "their own" more general results. That is, complicated uncertainty structure can be "overbounded" by the interval one and this new family is sequentially tested. Unfortunately, this method brings into the analysis certain degree of conservatism due to ignoring of mutual dependencies among coefficients in the original family. As a result, robust stability is investigated only with sufficient (i.e. stronger) and not necessary and sufficient condition.

The preceding consideration is demonstrated on example from (Barmish 1994). The family of polynomials (with multilinear uncertainty structure) is described by:

$$
\begin{aligned}
& p(s, q)=s^{4}+\left(5+0.2 q_{1} q_{2}+0.1 q_{1}-0.1 q_{2}\right) s^{3}+ \\
& +\left(6+3 q_{1} q_{2}-4 q_{2}\right) s^{2}+\left(6+6 q_{1}-8 q_{2}\right) s+ \\
& +\left(0.5-3 q_{1} q_{2}\right)
\end{aligned}
$$

and uncertainty bound $\left|q_{i}\right| \leq 0.25$ for $i=1,2$. The objective is to determine whether $p(s, q)$ is robustly stable. New bounds can be computed as:

$$
\begin{aligned}
& \bar{q}_{0}^{-}=\min _{q \in Q} a_{0}(q)=\min _{-0.25 \leq q_{i} \leq 0.25}\left(0.5-3 q_{1} q_{2}\right)=0.3125 \\
& \bar{q}_{0}^{+}=\max _{q \in Q} a_{0}(q)=\max _{-0.25 \leq q_{i} \leq 0.25}\left(0.5-3 q_{1} q_{2}\right)=0.6875 \\
& \bar{q}_{1}^{-}=\min _{q \in Q} a_{1}(q)=\min _{-0.25 \leq q_{i} \leq 0.25}\left(6+6 q_{1}-8 q_{2}\right)=2.5 \\
& \bar{q}_{1}^{+}=\max _{q \in Q} a_{1}(q)=\max _{-0.25 \leq q_{i} \leq 0.25}\left(6+6 q_{1}-8 q_{2}\right)=9.5
\end{aligned}
$$

Analogical computations yield to the coefficients $\bar{q}_{2}^{-}=4.8125, \bar{q}_{2}^{+}=7.1875, \bar{q}_{3}^{-}=4.9475, \bar{q}_{3}^{+}=5.0375$. The overbounding interval family of polynomials is given by:

$$
\begin{aligned}
& \bar{p}(s, \bar{q})=s^{4}+[4.9475,5.0375] s^{3}+ \\
& +[4.8125,7.1275] s^{2}+[2.5,9.5] s+ \\
& +[0.3125,0.6875]
\end{aligned}
$$

By applying the Kharitonov theorem or graphical test, it is straightforward to verify that the family (17) is robustly stable. Therefore, it can be concluded that the original family (15) must also be robustly stable. Thus, the overbounding method has been successful in this case.

However, one has to be careful here. As it was adumbrated hereinbefore, if the new overbounding family was not robustly stable, it could not to be decided about the authentic family, i.e. although the Kharitonov rectangles would cover the origin of the complex plane, the value sets of the original family would not necessarily have to include it. This is illustrated in next example (Šebek 2009), where the polynomial family (with affine linear uncertainty structure) is given by:

$$
\begin{aligned}
& p(s, q)=s^{4}+\left(2 q_{2}+1\right) s^{3}+\left(2 q_{1}-q_{2}+4\right) s^{2}+ \\
& +\left(q_{2}+1\right) s+\left(q_{1}-2 q_{2}+2\right)
\end{aligned}
$$

with uncertainty bounds $q_{1} \in\langle-0.5,2\rangle, q_{2} \in\langle-0.3,0.3\rangle$. The overbounding interval polynomial is then:

$$
\begin{aligned}
& \bar{p}(s, \bar{q})=s^{4}+[0.4,1.6] s^{3}+[2.7,8.3] s^{2}+ \\
& +[0.7,1.3] s+[0.9,4.6]
\end{aligned}
$$

As can be easily verified by the Kharitonov theorem, the overbounding family (19) is not robustly stable so it can not be said anything about robust stability of (18) and it has to be tested with the assistance of a more advanced method, i.e. the overbounding method is unsuccessful here. Apropos, the family (18) is robustly stable in fact.

\section{CONCLUSION}

The principal aim of the contribution has been to present the advantages and restrictions which arise during use of interval uncertainty. In addition to this, it has clarified the origin of uncertainties and it has shown their possible integration into mathematical model of controlled system. The paper has described the main tools of robust stability analysis, such as Kharitonov theorem, the value set concept and the zero exclusion condition, using the necessary theoretical background and consequently some illustrative examples. The final part is dedicated to usefullness of the methods also for, in practice very common, structures which are more complicated than only interval ones.

\section{ACKNOWLEDGEMENT}

The work was supported by the Ministry of Education, Youth and Sports of the Czech Republic under Research Plan No. MSM 7088352102. This assistance is very gratefully acknowledged.

\section{REFERENCES}

Anagnost, J.J.; C.A. Desoer and R.J. Minnichelli. 1989. "Generalized Nyquist test for robust stability: Frequency domain generalizations of Kharitonov's theorem". In Robustness in Identification and Control (M. Milanese; R. Tempo and A. Vicino, Eds.), New York, Plenum Press, 79-96.

Anderson, B.D.O.; E.I. Jury and M. Mansour. 1987. "On robust Hurwitz polynomials". IEEE Transactions on Automatic Control, Vol. 32, No. 10, 909-913.

Barmish, B.R. 1983. "Invariance of the strict Hurwitz property for polynomials with perturbed coefficients". In Proceedings of the IEEE Conference on Decision and Control (San Antonio, Texas). 
Barmish, B.R. 1989. “A generalization of Kharitonov's four polynomial concept for robust stability problems with linearly dependent coefficient perturbations". IEEE Transactions on Automatic Control, Vol. 34, No. 2, 157165.

Barmish, B.R. 1994. New Tools for Robustness of Linear Systems. Macmillan, New York, USA.

Bialas, S. 1983. "A necessary and sufficient condition for stability of interval matrices". International Journal of Control, Vol. 37, No. 4, 717-722.

Dasgupta, S. 1988. "Kharitonov's theorem revisited". Systems \& Control Letters, Vol. 11, No. 5, 381-384.

Kharitonov, V.L. 1979. "Asymptotic stability of an equilibrium position of a family of systems of linear differential equations". Differential Equations, Vol. 14, 1483-1485.

Kučera, V. 2001. "Robust Controllers (Robustní regulátory)". Automa, Vol. 7, No. 6, 43-45. (In Czech).

Matušù, R. and R. Prokop. 2008. "Robust stability of systems with parametric uncertainty". Archives of Control Sciences, Vol. 18(LIV), No. 1, 73-87.

Mikhailov, A.W. 1938. "Method of harmonic analysis in control theory". Avtomatika i Telemekhanika, No. 3, 27 81. (In Russian).

Minnichelli, R.J.; J.J. Anagost and C.A. Desoer. 1989. “An elementary proof of Kharitonov's theorem with extensions". IEEE Transactions on Automatic Control, Vol. 34, No. 9, 995-998.

Polyx. "The Polynomial Toolbox". [online]. [cit. 15-02-2009]. Available from URL: $<$ http://www.polyx.com/>.

Šebek, M. "Robust control (Robustní řízení)". [online]. PDF slides for course "Robust Control", Czech Technical University in Prague. [cit. 15-02-2009]. Available from URL: <http://dce.felk.cvut.cz/ror/prednasky_sebek.html>. (In Czech)

Šebek, M.; M. Hromčík and J. Ježek. 2000. "Polynomial toolbox 2.5 and systems with parametric uncertainties". In Proceedings of 3rd IFAC Symposium on Robust Control Design (Prague, Czech Republic).

\section{AUTHOR BIOGRAPHIES}

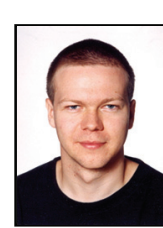

RADEK MATUŠŮ was born in Zlín, Czech Republic in 1978. He is a Researcher at Faculty of Applied Informatics of Tomas Bata University in Zlín. He graduated from Faculty of Technology of the same university with an MSc in Automation and Control Engineering in 2002 and he received a PhD in Technical Cybernetics from Faculty of Applied Informatics in 2007. He worked as a Lecturer from 2004 to 2006. The main fields of his professional interest include robust systems and application of algebraic methods to control design. His e-mail address is: rmatusu@fai.utb.cz and his web-page can be found at: http: //zamestnanci.fai.utb.cz/ matusu/.



ROMAN PROKOP was born in Hodonin, Czech Republic in 1952. He graduated in Cybernetics from the Czech Technical University in Prague in 1976. He received post graduate diploma in 1983 from the Slovak Technical University. Since 1995 he has been at Tomas Bata University in Zlín, where he presently holds the position of full professor of the Department of Automation and Control Engineering and a vice-rector of the university. His research activities include algebraic methods in control theory, robust and adaptive control, autotuning and optimization techniques. His e-mail address is: prokop@fai.utb.cz.

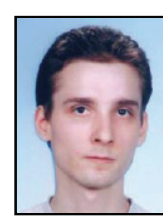

JIŘí KORBEL was born in Zlín, Czech Republic. He studied automatic control and informatics at the Tomas Bata University and graduated in 2004, now he is an post-graduate student and assistant at the Faculty of Applied Informatics in Zlín. His research activities include autotuning principles, algebraic and polynomial syntheses and modeling and simulations. His e-mail address is: korbel@fai.utb.cz. 